\title{
Relação entre imagem corporal, atitudes para transtornos alimentares e estado nutricional em universitárias da área da saúde
}

\author{
Relationship between body image, attitudes towards eating disorders \\ and nutritional status in university students in the health area \\ Amanda Luisa Kessler', Fabiana Assmann Poll²
}

\section{RESUMO}

Objetivo: Avaliar a relação entre a insatisfação da imagem corporal, atitudes para transtornos alimentares e o estado nutricional em universitárias da área da saúde. Métodos: Estudo descritivo, transversal e quantitativo com 225 universitárias matriculadas em oito cursos da área da saúde. Para obtenção dos dados, utilizaram-se três instrumentos autoaplicáveis: o Body Shape Questionnaire (BSQ), o Eating Attitudes Test (EAT-26) e um questionário construído pelas pesquisadoras contendo os dados autorreferidos de altura, peso atual e peso desejado, curso e idade. O estado nutricional foi estabelecido segundo o cálculo do índice de massa corporal. Para análise dos dados, aplicou-se o teste qui-quadrado de Pearson, adotando-se o nível de significância de 5\%. Resultados: A média de idade foi de 22,65 anos e a de IMC, de 23,05 $\mathrm{kg} / \mathrm{m}^{2}$, estando a maioria das universitárias em eutrofia (69,8\%). Quanto ao peso desejado, $74,7 \%$ gostariam de pesar menos; dessas, 64,9\% eram eutróficas. Conforme o BSQ, 51,1\% das universitárias tiveram algum grau de insatisfação corporal, e a prevalência de atitudes indicativas de transtornos alimentares, de acordo com o EAT-26, foi de 21,8\%. Houve associação estatisticamente significativa entre: IMC e BSQ ( $p<0,001)$; IMC e EAT-26 ( $p<0,005)$; BSQ e EAT-26 ( $p<0,001)$. Ao relacionar a insatisfação da imagem corporal e atitudes de risco para transtornos alimentares, percebeu-se que $87,75 \%$ das universitárias com EAT positivo também apresentaram algum grau de insatisfação corporal. Conclusão: Evidenciou-se a existência de relação entre a insatisfação com a imagem corporal, atitudes de risco para transtornos alimentares e estado nutricional de universitárias da área da saúde.

\section{ABSTRACT}

Objective: To evaluate the relationship between body image dissatisfaction, attitudes towards eating disorders and nutritional status in university students of health area. Methods: Descriptive, transversal and quantitative study with 225 university students registered in eight health courses. To obtain the data, three self-administered instruments were used: the Body Shape Questionnaire (BSQ), the Eating Attitudes Test (EAT-26), and a questionnaire built by the researchers, containing the self-reported data of height, current and desired weight,

1 Universidade de Santa Cruz do Sul (UNISC), Santa Cruz do Sul, RS, Brasil.

2 Universidade de Santa Cruz do Sul (UNISC), Departamento de Educação Física e Saúde, Santa Cruz do Sul, RS, Brasil. 


\section{Keywords}

Body image, feeding and eating disorders, nutritional status, students. course and age. The nutritional status was established according to the calculation of body mass index (BMI). For the data analysis, the Pearson chi-square test was applied, adopting the significance level of 5\%. Results: The mean age was 22.65 years and the BMI was 23.05 $\mathrm{kg} / \mathrm{m}^{2}$, with the majority of university students in eutrophy (69.8\%). Regarding the desired weight, $74.7 \%$ would like to weigh less, of which $64.9 \%$ were eutrophic. According to the BSQ, $51.1 \%$ of university students had some degree of body dissatisfaction and the prevalence of attitudes indicative of eating disorders, according to the EAT-26, it was 21.8\%. There was a statistically significant association between: BMI and BSQ ( $p<0.001)$; BMI and EAT-26 ( $p<$ $0.005)$; BSQ and EAT-26 ( $p<0.001)$. When correlating the dissatisfaction of body image and attitudes of risk for eating disorders, it is noticed that $87.75 \%$ of university students with positive EAT also presented some degree of body dissatisfaction. Conclusion: It was evidenced the existence of relation between the body image dissatisfaction, attitudes of risk for eating disorders and nutritional status of university students of healtharea.

\section{INTRODUÇÃO}

Há atualmente uma crescente busca pela maior compreensão do comportamento alimentar de indivíduos e coletividades, uma vez que ele depende do modo como cada ser interage e se relaciona com os alimentos' ${ }^{1}$. A alimentação é uma prática permanente e essencial para a sobrevivência humana, sendo definida como um fenômeno complexo que abrange aspectos psicológicos, fisiológicos e socioculturais².

Nos transtornos alimentares, essas práticas estão altamente comprometidas, pois são caracterizados por consumo alimentar irregular, compulsão e obsessão pela comi$\mathrm{da}$, dietas restritivas e comportamentos purgativos ${ }^{2,3}$. Eles podem ser definidos como síndromes comportamentais de etiologia multifatorial, que envolvem fatores genéticos, psicológicos e/ou socioculturais ${ }^{4-6}$. Entre os transtornos do comportamento alimentar mais comuns, estão a anorexia e a bulimia nervosas e o transtorno da compulsão alimentar periódica ${ }^{7-9}$.

O estudo dos transtornos alimentares tem despertado cada vez mais o interesse de profissionais da saúde, por apresentarem elevado índice de morbidades associadas e por influenciarem o estado nutricional e o metabolismo dos indivíduos ${ }^{10,11}$. Sabe-se que a prevalência deles vem aumentando com o passar das décadas, afetando principalmente mulheres, adolescentes e jovens, que representam fases da vida em que ocorrem diversas mudanças $5^{5,7-9,11-16}$.

Ainda, é referida na literatura alta prevalência de transtornos alimentares em estudantes de graduações na área da saúde, em que a aparência física e a boa forma são tidas como aspectos relevantes e associados ao bom desempenho e sucesso profissional ${ }^{12,17-23}$.

A partir de estudos recentes, observa-se que os transtornos alimentares, se não tratados, tornam-se círculos viciosos que podem impactar significativamente a vida social do indivíduo, uma vez que ele acaba se isolando do convívio com outras pessoas por causa da sua preocupação exacerbada com alimentação e imagem corporal ${ }^{24,25}$.
Nesse contexto, a imagem corporal é definida pela capacidade que cada ser possui de interpretação mental de seu próprio corpo, ou seja, é a forma como o enxergamos e vivenciamos $^{5,9}$. Esse conceito é algo muito particular e interno e que proporciona a cada um sensações, pensamentos e percepções distintas quanto ao tamanho e forma do próprio corpo ${ }^{26}$.

Salienta-se que a vida universitária pode ser vista como um período peculiar em termos de nutrição, haja vista que muitas vezes a alimentação é deixada em segundo plano, não ocupando a devida importância que deveria 16,21,22. Esse fato decorre das intensas mudanças que esse período implica, bem como do acúmulo de tarefas, responsabilidades e novo estilo de vida adotado ${ }^{27}$.

Tendo em vista que essa é uma fase em que hábitos de vida são adquiridos, é importante que se tenha maior atenção com esse grupo de indivíduos, pois normalmente esses hábitos costumam se perpetuar na fase adulta e podem estar associados, futuramente, com má qualidade de vidaa,28.

Sendo assim, o objetivo do estudo foi avaliar a relação entre insatisfação da imagem corporal, atitudes para transtornos alimentares e estado nutricional em universitárias da área da saúde.

\section{MÉTODOS}

\section{Amostra}

Trata-se de um estudo descritivo, de caráter transversal e abordagem quantitativa, com 225 universitárias matriculadas em oito cursos da área da saúde da Universidade de Santa Cruz do Sul/RS (UNISC), sendo eles: Educação Física (licenciatura e bacharelado), Enfermagem, Farmácia, Fisioterapia, Graduação Tecnológica em Estética e Cosmética, Medicina, Nutrição e Odontologia.

Para a delimitação da amostra, optou-se pelo modelo probabilístico, utilizando-se da amostra casual estratificada. Dessa forma, a população foi composta por 10\% das estu- 
dantes de cada curso conforme o número de alunos matriculados neles. Os critérios de inclusão para participar do estudo foram: a) aceitar participar voluntariamente da pesquisa mediante assinatura do Termo de Consentimento Livre e Esclarecido; b) ter idade maior ou igual a 18 anos; c) ser do gênero feminino; d) estar regularmente matriculada entre o quarto e o sexto semestre dos cursos de graduação.

O presente estudo cumpre os princípios éticos que orienta e regulamenta pesquisas envolvendo seres humanos, de acordo com a Resolução no 466, de 12 de dezembro de 2012, do Conselho Nacional de Saúde ${ }^{29}$. Para isso, o Termo de Consentimento Livre e Esclarecido da pesquisa foi apresentado aos sujeitos no momento da coleta de dados para sua leitura e concordância de sua participação livre e voluntária. O presente estudo também foi aprovado pelo Comitê de Ética em Pesquisa da UNISC, sob o protocolo no 1.876.615.

\section{Procedimentos}

Após a apresentação da pesquisa e do consentimento dos docentes e das coordenações dos cursos, a coleta de dados ocorreu durante as aulas curriculares, em datas e horários previamente agendados, dentro das dependências da universidade.

Para obtenção dos dados, utilizaram-se três instrumentos autoaplicáveis: o Body Shape Questionnaire (BSQ), o Eating Attitudes Test (EAT-26), bem como um questionário com informações pessoais e perfil nutricional construído pelas pesquisadoras, que abrangeu as seguintes questões: curso, idade, peso e altura autorreferidos e peso desejado. Em relação ao peso e à altura autorreferidos, eles têm sido utilizados em muitos estudos, devido tanto à sua praticidade quanto à alta concordância entre a informação coletada e a aferição ${ }^{30,31}$.

A avaliação do estado nutricional atual foi feita por meio do cálculo do índice de massa corporal (IMC), classificado de acordo com a Organização Mundial da Saúde (OMS) 32 .

\section{Instrumentos}

\section{Body Shape Questionnaire (BSQ)}

Questionário de autopreenchimento, desenvolvido por Cooper et al. ${ }^{33}$, traduzido por Cordás e Neves $^{34}$ e validado para a população brasileira por Di Pietro e Silveira ${ }^{35}$. É formado por 34 questões em escala do tipo Likert de seis pontos, que variam de 1 = nunca até $6=$ sempre. Trata-se de um instrumento validado para universitários de ambos os sexos que busca avaliar a frequência da preocupação, descontentamento e insatisfação com a imagem corporal e o peso. É um instrumento que propicia avaliação constante e descritiva dos distúrbios da imagem corporal, sendo possível que se mensure seu papel no desenvolvimento, manutenção e resposta ao tratamento de distúrbios como a anorexia e a bulimia nervosa ${ }^{36,37}$.

Para a classificação dos resultados obtidos com a aplicação do $B S Q$, soma-se o total de pontos adquiridos con- forme a resposta assinalada e classifica-se a insatisfação da imagem corporal, conforme o nível de descontentamento e preocupação, sendo: Ausência de insatisfação: $\leq 80$; Leve insatisfação: $\geq 81$ e $\leq 110$; Moderada insatisfação: $\geq 111$ e < 140; Grave insatisfação: $\geq 140$. Os pontos de corte adotados seguem os estabelecidos por Di Pietro e Silveira ${ }^{35}$.

\section{Eating Attitudes Test (EAT-26)}

Desenvolvido por Garner et al..$^{38}$ e traduzido para o português por dois autores: Bighetti et al. ${ }^{39}$, que avaliaram sua validade interna quando aplicado a meninas adolescentes, e Nunes et al. ${ }^{40}$, que validaram o instrumento. Ainda, Magalhães e Mendonça ${ }^{41}$ encontraram boa reprodutibilidade do instrumento em jovens universitárias.

O questionário contém 26 itens, em escala do tipo Likert de seis pontos, que variam de $0=$ nunca até $6=$ sempre. O EAT-26 é reconhecido internacionalmente e tornou-se um dos testes mais aplicados para identificar a presença de padrões alimentares anormais indicativos de transtornos alimentares ${ }^{42,43}$.

Para a classificação do EAT-26, são atribuídos pontos de 0 a 3, de acordo com as respostas marcadas no questionário, sendo: sempre $=3$ pontos; muitas vezes $=2$ pontos; às vezes $=1$ ponto; poucas vezes $=0$ ponto; quase nunca $=0$ ponto e nunca $=0$ ponto. A única questão que apresenta pontuação em ordem invertida é a número $4^{44,45}$. Um resultado maior que 21 pontos representa teste EAT positivo, sendo o entrevistado classificado em risco para o desenvolvimento de transtornos alimentares. Um escore abaixo de 21 pontos indica EAT negativo, estando o indivíduo classificado com padrão alimentar em normalidade ${ }^{44}$.

\section{Análise dos dados}

Todos os dados obtidos nos três instrumentos da pesquisa foram analisados estatisticamente no software SPSS 20.0. Os indivíduos foram agrupados de acordo com a classificação de sua imagem corporal e da presença de atitudes relacionadas aos transtornos alimentares. As análises foram feitas a partir da amostra total, sem diferenciar os cursos envolvidos.

As variáveis foram descritas em medidas de tendência central (média) e de dispersão (desvio-padrão), e as prevalências foram descritas em frequência absoluta e relativa. Foi realizada comparação descritiva do IMC com as variáveis de insatisfação corporal, atitudes alimentares e peso desejado. Utilizou-se o teste qui-quadrado de Pearson para verificar as associações entre as variáveis IMC, BSQ e EAT-26. O nível de significância adotado foi de 5\%.

\section{RESULTADOS}

Participaram do estudo 225 acadêmicas de oito cursos da área da saúde, sendo: 50 universitárias do curso de Educação 
Física (licenciatura e bacharelado), 35 de Enfermagem, 19 de Farmácia, 28 de Fisioterapia, 8 de Graduação Tecnológica em Estética e Cosmética, 38 de Medicina, 21 de Nutrição e 26 universitárias do curso de Odontologia. As idades variaram entre 18 e 48 anos, com média de idade de 22,65 \pm 4,77 anos, e 48\% tinham entre 21 e 25 anos.

As medidas antropométricas autorreferidas apontaram média de peso de $62 \pm 10,14 \mathrm{~kg}$ e 1,64 \pm 0,63m de altura e de IMC de 23,05 $\pm 3,44 \mathrm{~kg} / \mathrm{m}^{2}$. Em relação à classificação do estado nutricional, obteve-se que $69,8 \%$ das estudantes encontravam-se em eutrofia, 20,9\% em sobrepeso, 4,4\% em baixo peso, $4 \%$ em obesidade grau l e 0,9\% em obesidade grau II.

Quando questionadas sobre o peso desejado, $74,7 \%$ das acadêmicas refeririam que gostariam de pesar menos, em média 4,62 \pm 6,39 kg; dessas, 64,9\% eram eutróficas.

Em relação ao $\mathrm{BSQ}$, o instrumento indicou escore médio de 87,05 \pm 33,23 pontos, com mínimo de 34 e máximo de 198 pontos, e constatou-se que $51,1 \%$ das universitárias tiveram algum grau de insatisfação corporal (Tabela 1).

Tabela 1. Avaliação da insatisfação da imagem corporal das universitárias, segundo o BSQ $(n=225)$

\begin{tabular}{lcc}
\hline Classificação BSQ & $\mathbf{n}$ & $\%$ \\
\hline Ausência de insatisfação & 110 & 48,9 \\
Leve insatisfação & 67 & 29,8 \\
Moderada insatisfação & 29 & 12,9 \\
Grave insatisfação & 19 & 8,4 \\
Total & 225 & 100,0 \\
\hline
\end{tabular}

n: frequência absoluta; \%: frequência relativa.

A prevalência geral de atitudes indicativas de transtornos alimentares, de acordo com o EAT-26, foi de 21,8\%, com escore médio de 15,5 \pm 8,47 pontos, escore mínimo de 0 e escore máximo de 47 pontos (Tabela 2).

Tabela 2. Avaliação de atitudes para transtornos alimentares das universitárias, segundo o EAT- $26(n=225)$

\begin{tabular}{lcc}
\hline Classificação EAT & $\mathbf{n}$ & $\%$ \\
\hline EAT positivo & 49 & 21,8 \\
EAT negativo & 176 & 78,2 \\
Total & 225 & 100,0 \\
\hline
\end{tabular}

EAT positivo: > 21 pontos. n: frequência absoluta; \%: frequência relativa.

Foi observada associação estatisticamente significativa entre IMC e BSQ $(p<0,001)$ e entre IMC e EAT-26 $(p<0,005)$. Em relação ao IMC e BSQ, nota-se que estudantes com peso adequado foram mais insatisfeitos com a imagem corporal do que aqueles com IMC baixo ou em sobrepeso e obesidade. Destaca-se, no entanto, que a insatisfação moderada $(n=15)$ e grave $(n=11)$ com a imagem corporal foi mais prevalente naquelas estudantes com algum grau de excesso de peso, somando-se nesse as classificações de sobrepeso, obesidade grau l e obesidade grau II (Tabela 3).

Referente ao cruzamento das classificações do IMC e EAT-26, a pontuação positiva do EAT -26 foi mais prevalente em universitárias com peso eutrófico. Porém, quando somadas as classificações para sobrepeso, obesidade grau l e obesidade grau II, os dados mostraram-se semelhantes aos dos eutróficos ( $n=23)$ (Tabela 3).

Ao relacionar a insatisfação da imagem corporal e atitudes de risco para transtornos alimentares, percebe-se que $87,75 \%$ das universitárias com EAT positivo $(n=43)$ também apresentaram algum grau de insatisfação corporal (Tabela 4).

\section{DISCUSSÃO}

Este estudo avaliou a relação entre a insatisfação da imagem corporal, atitudes para transtornos alimentares e o estado nutricional de universitárias da área da saúde.

Os transtornos do comportamento alimentar tiveram sua incidência mundial praticamente dobrada nos últimos 20 anos, demonstrando que há crescente preocupação da população com sua imagem corporal ${ }^{10}$. Nesse sentido, estudos relacionados à imagem corporal vêm crescendo significativamente, tendo em vista que sua percepção influencia nos hábitos, comportamentos e consumo alimentar. Isso posto, observa-se que, quando o indivíduo tem uma interpretação negativa da aparência, com sentimentos de depreciação ou preocupação extrema com a forma física, ele utiliza atitudes inadequadas direcionadas a manutenção ou alcance de um corpo desejado. Essas atitudes, por sua vez, ocasionam prejuízo da qualidade de vida e podem ser sintomas de primeira ordem no desenvolvimento de transtornos alimentares ${ }^{43,46}$.

No presente estudo, observou-se que a maioria das estudantes se encontrava com estado nutricional adequado, corroborando outros estudos realizados com esse públi$\mathrm{co}^{5,24,26,47-49}$. Relativamente ao peso desejado, percebeu-se grande prevalência de jovens descontentes com o peso atual e com anseio de perda de peso, e a prevalência desse desejo foi maior entre as eutróficas, ratificando o estudo feito por Bosi et al..24, em que 88,3\% das estudantes do curso de Medicina com IMC normal indicaram desejo de perda de peso, em média $3,3 \mathrm{~kg}$, similar ao valor encontrado nesse estudo.

Diante disso, é possível evidenciar um notável descontentamento com a forma física e desejo de perda de peso, mesmo naquelas universitárias com peso adequado e saudável em relação à altura e à idade. Essa situação gera reflexões sobre o conceito de saúde e aparência física veiculado atualmente, que é de um corpo magro e musculoso, o que enaltece a valorização da imagem corporal e direciona a busca por esse padrão estético que nem sempre é saudável e, em muitos casos, impossível de se atingir, uma vez que não se consideram as diferenças sociais e biológicas de cada um ${ }^{50,51}$. 
Tabela 3. Relação entre a classificação do IMC e a classificação do BSQ e do EAT-26 ( $n=225)$

\begin{tabular}{|c|c|c|c|c|c|c|c|}
\hline \multicolumn{8}{|c|}{ Classificação IMC } \\
\hline & Baixo peso (n) & Eutrofia (n) & Sobrepeso (n) & Obesidade grau I (n) & Obesidade grau II (n) & Total (n) & $p^{*}$ \\
\hline \multicolumn{8}{|l|}{ Classificação BSQ } \\
\hline Ausência de insatisfação & 9 & 87 & 11 & 3 & 0 & 110 & \\
\hline Leve insatisfação & 1 & 48 & 16 & 2 & 0 & 67 & \\
\hline Moderada insatisfação & 0 & 14 & 15 & 0 & 0 & 29 & $<0,001$ \\
\hline Grave insatisfação & 0 & 8 & 5 & 4 & 2 & 19 & \\
\hline \multicolumn{8}{|l|}{ Classificação EAT } \\
\hline EAT positivo & 2 & 24 & 18 & 4 & 1 & 49 & $<0,005$ \\
\hline EAT negativo & 8 & 133 & 29 & 5 & 1 & 176 & \\
\hline Total (n) & 10 & 157 & 47 & 9 & 2 & 225 & \\
\hline
\end{tabular}

EAT positivo: $>21$ pontos. ${ }^{*}$ Teste qui-quadrado de Pearson significativo para $p<0,05$.

Tabela 4. Comparação entre a insatisfação da imagem corporal e atitudes para transtornos alimentares das universitárias

\begin{tabular}{|c|c|c|c|c|}
\hline \multirow{2}{*}{ Classificação BSQ* } & \multicolumn{3}{|c|}{ Classificação EAT-26* } & \multirow[b]{2}{*}{$p^{*}$} \\
\hline & EAT positivo (n) & EAT negativo (n) & Total (n) & \\
\hline Ausência de insatisfação & 6 & 104 & 110 & \\
\hline Leve insatisfação & 14 & 53 & 67 & \\
\hline Moderada insatisfação & 13 & 16 & 29 & $<0,001$ \\
\hline Grave insatisfação & 16 & 3 & 19 & \\
\hline Total & 49 & 176 & 225 & \\
\hline
\end{tabular}

EAT positivo: $>21$ pontos. * Teste qui-quadrado de Pearson significativo para $p<0,05$.

Em relação ao BSQ, verificou-se que $51,1 \%$ das acadêmicas participantes do estudo apresentaram algum grau de insatisfação com a imagem corporal, somando-se os escores para leve, moderada e grave insatisfação, resultado semeIhante a outros estudos que aplicaram o mesmo instrumento em universitárias ${ }^{24-26}$. Essa insatisfação, quando comparada ao IMC, demostrou-se maior nas estudantes com estado nutricional normal, similar ao estudo de Silva et al. ${ }^{25}$, realizado com estudantes do curso de Nutrição, em que a maioria das estudantes com BSQ positivo para insatisfação corporal estava em eutrofia. Presume-se, assim, grande participação do imaginário da autoimagem quanto ao desejo de atingir cada vez mais os padrões de beleza impostos e divulgados amplamente pela sociedade e mídia atuais ${ }^{5}$.

Lira et al. ${ }^{15}$, ao analisar a influência da mídia e redes sociais com a insatisfação da imagem corporal, por meio da Escala de Atitudes Socioculturais em Relação à Aparência (SATAQ-3), encontrou uma relação verdadeira entre as variáveis, uma vez que meninas que desejavam ter silhuetas menores demostraram maior aceitação das mensagens da mídia relacionados ao ideal estético e físico corporal.

Contudo, salienta-se que no presente estudo os escores do BSQ para moderada e grave insatisfação corporal estiveram maiores nas estudantes com sobrepeso e obesidade, corroborando os resultados encontrados em pesquisas semelhantes, em que o grau de insatisfação com a imagem corporal, de acordo com o BSQ, foi maior em universitárias com excesso de peso 24,31,22,52,53. Diante disso, pode-se notar que estar acima do peso, para muitas jovens, implica infelicidade e baixa autoestima, com constante desejo de emagrecimento, a fim de se encaixarem numa norma social que cultua a magreza ${ }^{15}$.

Alvarenga et al..$^{49}$, ao utilizarem a Escala de Silhuetas de Stunkard, também demonstraram que universitárias em todas as classificações do IMC desejavam ter uma silhueta menor, evidenciando insatisfação corporal independentemente do estado nutricional. É importante ressaltar que essa percepção negativa da imagem corporal pode ser totalmente falsa e imaginária ou estar baseada em alterações sutis da aparência. Isso, por sua vez, pode conduzir a uma situação de insatisfação ou distorção de como o indivíduo percebe seu corpo, associada à adoção de atitudes alimentares inadequadas ${ }^{50,51}$.

A investigação por meio do EAT-26 demonstrou que $21,8 \%$ das acadêmicas apresentaram atitudes indicativas para desenvolvimento de transtornos alimentares, resultado próximo em relação ao de outros estudos com esse públi$\mathrm{co}^{12,23-25}$. A pontuação do EAT-26 positivo, quando cruzada 
com as classificações do IMC, mostrou-se maior nas estudantes com estado nutricional de eutrofia $(48,97 \%)$ em relação aos demais estados nutricionais. Esse dado condiz com a situação de indivíduos com bulimia nervosa, que, apesar da preocupação exagerada com o corpo, costumam estar com o peso normal, ou em alguns casos até levemente acima?. Reiterando ideias anteriormente discutidas, destaca-se que a distorção da imagem corporal leva a comportamentos purgativos como forma de "alívio de consciência" após a ingestão de grande quantidade de comida².

Entretanto, quando somados o sobrepeso e as obesidades grau I e II, caracterizando assim o excesso de peso (46,93\%), não houve diferença considerável entre as classificações do IMC. Kravchychyn et al. ${ }^{50}$, em estudo realizado com atletas do gênero feminino, também não encontraram disparidade do EAT-26 positivo com as classificações do IMC.

Ainda, nesse contexto, evidencia-se na literatura grande prevalência de transtornos alimentares em jovens com excesso de peso e histórico de depressão ${ }^{54}$, sendo os universitários da área da saúde os que apresentam maior preocupação com a aparência física, associado, principalmente, ao desejo de êxito na carreira, visto que existe um conceito que considera que profissionais da saúde devam ser exemplo de saúde e boa forma corporal17.22,26,31. Somam-se a essa realidade, ainda, os casos em que a comida é tida como forma de recompensa de condições de estresse físico e mental, indicando sinais de compulsão alimentar ${ }^{55}$.

A partir da comparação entre os resultados dos instrumentos BSQ e EAT-26, no corrente estudo, observou-se uma associação estatisticamente significativa entre as variáveis ( $p<0,001)$, estando a insatisfação com a imagem corporal relacionada com a presença de atitudes alimentares de riscos para o desenvolvimento de transtornos alimentares. Ademais, evidenciou-se maior prevalência de universitárias com grave insatisfação associada ao EAT-26 positivo, similar aos resultados de outros estudos com estudantes universitários $^{22,26,32,56}$.

Esse fato pode decorrer devido aos modelos estabelecidos socialmente, no tocante à aparência física, pois estes desempenham influência expressiva na construção da imagem corporal, particularmente de mulheres jovens, afetando a percepção em relação ao corpo e podendo prever um início de sintomas indicativos de transtornos alimentares nesse público, a fim de se adequarem aos modelos estabelecidos pela sociedade e mídia atuais ${ }^{57,58}$.

\section{CONCLUSÃO}

Os dados da presente pesquisa permitem concluir que há relação entre a insatisfação da imagem corporal, atitudes de risco para desenvolvimento de transtornos alimentares e estado nutricional de universitárias da área da saúde.
A insatisfação com a imagem corporal e a presença de atitudes alimentares de risco foram mais prevalentes naquelas estudantes com estado nutricional em eutrofia. Contudo, as universitárias com algum grau de excesso de peso apresentaram maior prevalência de insatisfação moderada ou grave com a imagem corporal, de acordo com o BSQ. Do mesmo modo, referente ao EAT-26, quando somadas as classificações para sobrepeso e obesidades graus I e II, o escore positivo para atitudes indicativas de transtornos alimentares mostrou-se semelhante ao dos eutróficos.

Ressalta-se ainda a importância de mais estudos sobre a prevalência de distúrbios alimentares, especialmente em grupos específicos como estudantes de cursos associados a alimentação e cuidados com a saúde, o que possibilitaria explorar causas ligadas aos sintomas de transtornos alimentares, suas possíveis consequências na formação e posterior atuação profissional e, também, meios de prevenção e conscientização acerca da forte pressão sociocultural existente, em que se estabelece um ideal de corpo que, muitas vezes, se sobrepõe aos princípios da saúde e bem-estar.

\section{CONTRIBUIÇÕES INDIVIDUAIS}

Amanda Luisa Kessler - Foi responsável pela concepção e desenho do estudo, coleta, organização, análise e interpretação dos dados, revisão da literatura e escrita do artigo.

Fabiana Assmann Poll - Foi responsável pela concepção e desenho do estudo, interpretação dos resultados e revisão crítica do conteúdo do artigo, e aprovou a versão final a ser publicada.

\section{CONFLITOS DE INTERESSE}

Os autores declaram não haver conflitos de interesse.

\section{AGRADECIMENTOS}

Os autores agradecem aos docentes e coordenadores dos cursos pesquisados, por possibilitarem e facilitarem a coleta de dados durante as aulas curriculares, e também às participantes do estudo, pela colaboração.

\section{REFERÊNCIAS}

1. Vaz DSS, Bennemann RM. Comportamento alimentar e hábito alimentar: uma revisão. Uningá Rev. 2014;20(1):108-12.

2. Alvarenga M, Philippi ST. Estrutura, padrão, consumo e atitude alimentar: conceitos e aplicações nos transtornos alimentares. In: Alvarenga M, Scagliusi FB, Philippi ST, organizadores. Nutrição e transtornos alimentares: avaliação e tratamento. São Paulo: Manole; 2011. 
3. Kotait, MS, Barillari ML, Conti MA. Escalas de avaliação de comportamento alimentar. In: Cordás TA, Kachani AT. Nutrição em psiquiatria. Porto Alegre: Artmed; 2010.

4. Palma RFM, Santos JE, Ribeiro RPP. Evolução nutricional de pacientes com transtornos alimentares: experiência de 30 anos de um Hospital Universitário. Rev Nutr. 2013;26(6):669-78.

5. Bandeira YER, Mendes ALRF, Cavalcante ACM, Arruda SPM. Avaliação da imagem corporal de estudantes do curso de Nutrição de um centro universitário particular de Fortaleza. J Bras Psiquiatr. 2016;65(2):168-73

6. Fortes LS, Ferreira MEC, Costa PRF, Lira HAAS, Andrade J, Silva ALAG. Comparação do comportamento de risco para transtornos alimentares entre adolescentes atletas e não atletas. J Bras Psiquiatr. 2015;64(4):296-302.

7. Alves TCHS, Santana MLP, Silva RCR, Pinto EJ, Assis AMO. Fatores associados a sintomas de transtornos alimentares entre escolares da rede pública da cidade do Salvador, Bahia. J Bras Psiquiatr. 2012;61(2):55-63.

8. Silva $T A B$, Ximenes RCC, Holanda MA, Melo MG, Sougey EB, Couto GBL. Frequência de comportamentos alimentares inadequados e sua relação com a insatisfação corporal em adolescentes. J Bras Psiquiatr. 2012;61(3):154-8.

9. Fortes $L S$, Almeida SS, Ferreira MEC. Impacto de variáveis antropométricas sobre a insatisfação corporal e o comportamento alimentar em jovens atletas. J Bras Psiquiatr. 2012;61(4):235-41.

10. Uzunian $\mathrm{LG}$, Vitalle MSS. Habilidades sociais: fator de proteção contra transtornos alimentares em adolescentes. Ciênc Saúde Coletiva. 2015;20(11):3495-508.

11. Medeiros TH, Caputo EL, Domingues MR. Insatisfação corporal em frequentadoras de academia. J Bras Psiquiatr. 2017;66(1):38-44.

12. Carvalho PHB, Filgueiras JF, Neves CM, Coelho FD, Ferreira MEC. Checagem corporal, atitude alimentar inadequada e insatisfação com a imagem corporal de jovens universitários. J Bras Psiquiatr. 2013;62(2):108-14.

13. Fortes LS, Cipriani FM, Paes ST, Coelho FD, Ferreira MEC. Relação entre o estado de humor e os comportamentos alimentares de risco para os transtornos alimentares em adolescentes. J Bras Psiquiatr. 2016;65(2):155-60.

14. Leal GVS, Philippi ST, Polacow VO, Cordás TA, Alvarenga MS. 0 que é comportamento de risco para transtornos alimentares em adolescentes? J Bras Psiquiatr.2013;62(1):62-75.

15. Lira AG, Ganen AP, Lodi AS, Alvarenga MS. Uso de redes sociais, influência da mídia e insatisfação com a imagem corporal de adolescentes brasileiras. J Bras Psiquiatr. 2017;66(3):164-71.

16. Souza AC, Alvarenga MS. Insatisfação com a imagem corporal em estudantes universitários - Uma revisão integrativa. J Bras Psiquiatr. 2016;65(3):286-99.

17. Laus MF, Moreira RCM, Costa TMB. Diferenças na percepção da imagem corporal, no comportamento alimentar e no estado nutricional de universitárias das áreas de saúde e humanas. Rev Psiquiatr Rio Gd Sul. 2009;31(3):192-6.

18. Silva TAB, Vasconcelos FMN, Ximenes RCC, Sampaio TPA, Sougey EB. As terapias cognitivo-comportamentais no tratamento da bulimia nervosa: uma revisão. J Bras Psiquiatr. 2015;64(2):160-8

19. Claumann GS, Pereira EF, Inácio S, Santos MC, Martins AC, Pelegrini A. Body image satisfaction in freshmen college students in physical education courses. Rev Educ Fis UEM. 2014;25(4):575-83.

20. Frank R, Claumann GS, Pinto AA, Cordeiro PC, Felden EPG, Pelegrini A. Fatores associados à insatisfação com a imagem corporal em acadêmicos de Educação Física. J Bras Psiquiatr. 2016;65(2):161-7.

21. Martins BG, Silva WR, Campos JADB. Preocupação com a forma do corpo de graduandos de Farmácia-Bioquímica. J Bras Psiquiatr. 2015;64(1):32-9.

22. Miranda VPN, Filgueiras JF, Neves CM, Teixeira PC, Ferreira MEC. Insatisfação corporal em universitários de diferentes áreas de conhecimento. J Bras Psiquiatr. 2012;61(1):25-32.

23. Pereira LNG, Trevisol FS, Quevedo J, Jornada LK. Eating disorders among health science students at a university in southern Brazil. Rev Psiquiatr Rio Gd Sul. 2011;33(1):14-9.

24. Bosi MLM, Nogueira JAD, Uchimura KY, Luiz RR, Godoy MGC. Comportamento alimentar e imagem corporal entre estudantes de medicina. Rev Bras Educ Med. 2014;38(2):243-52.

25. Silva JD, Silva ABJ, Oliveira AVK, Nemer ASA. Influência do estado nutricional no risco para transtornos alimentares em estudantes de nutrição. Ciênc Saúde Coletiva. 2012;17(12):3399-4006.
26. Garcia CA, Castro TG, Soares RM. Comportamento alimentar e imagem corporal entre estudantes de nutrição de uma universidade pública de Porto Alegre - RS. Rev HCPA. 2010;30(3):219-24

27. Duarte FM, Almeida SDS, Martins KA. Alimentação fora do domicílio de universitários de alguns cursos da área da saúde de uma instituição privada. Mundo Saúde. 2013;37(3):288-98.

28. Moreira NWR, Castro LCV, Conceição LL, Duarte MS. Consumo a limentar, estado nutricional e risco de doença cardiovascular em universitários iniciantes e formandos de um curso de nutrição, Viçosa - MG. Rev APS. 2013;16(3):242-9.

29. Conselho Nacional de Saúde (Brasil). Resolução no 466, de 12 de dezembro de 2012. Diretrizes e Normas Regulamentadoras de Pesquisas Envolvendo Seres Humanos. Diário Oficial da União 12 dez. 2012, seção 1.

30. Bosi MLM, Luiz RG, Morgado CMC, Costa MLS, Carvalho RJ. Autopercepção da imagem corporal entre estudantes de nutrição: um estudo no município do Rio de Janeiro. J Bras Psiquiatr. 2006;55(2):108-13.

31. Reis JA, Silva Júnior CRR, Pinho L. Fatores associados ao risco de transtornos alimentares entre acadêmicos da área de saúde. Rev Gaúcha Enferm. 2014;35(2):73-8.

32. WHO. Obesity: preventing and managing the global epidemic. Geneva: World Health Organization: WHO; Technical Report Series;2000.

33. Cooper PJ, Taylor MJ, Cooper Z, Fairburn CG. The development and validation of the body shape questionnaire. Int J Eat Disord. 1987;6(4):485-94.

34. Cordás TA, Neves JEP. Escalas de avaliação de transtornos alimentares. Rev Psiquiatr Clin. 1999;26(1):41-7.

35. Di Pietro M, Silveira DX. Internal validity, dimensionality and performance of the Body Shape Questionnaire in a group of Brazilian college students. Rev Bras Psiquiatr. 2009;31(1):21-4

36. Amaral ACS. Adaptação transcultural do Sociocultural Atitudes Towards Appearance Questionnaire - 3 (SATAQ - 3) para a população brasileira [dissertação]. Juiz de Fora: Universidade Federal de Juiz de Fora; 2011.

37. Fortes $L S$, Filgueiras JF, Ferreira MEC. Comportamentos de risco para transtornos alimentares e sintomas depressivos: um estudo com jovens do sexo feminino de Juiz de Fora, Minas Gerais, Brasil. Cad Saúde Pública. 2014;30(11):2443-50.

38. Garner DM, Olmsted MP, Bohr Y, Garfinkel PE. The eating attitudes test: psychometric features and clinical correlates. Psychol Med. 1982;12(4):871-8.

39. Bighetti F, Santos (B, Santos JE, Ribeiro RPP. Tradução e validação do Eating Attitudes Test em adolescentes do sexo feminino de Ribeirão Preto, São Paulo. J Bras Psiquiatr. 2004;53(6):339-46.

40. Nunes MA, Camey S, Olinto MTA, Mari JJ. The validity and 4-year test-retest reliability of the Brazilian version of the Eating Attitudes Test-26. Braz J Med Biol Res. 2005;38(11):1655-62.

41. Magalhães VC, Mendonça GAS. Transtornos alimentares em universitárias: estudo de confiabilidade da versão brasileira de questionários autopreenchíveis. Rev Bras Epidemiol. 2005;8(3):236-45.

42. Kirsten VR, Fratton F, Porta NBD. Transtornos alimentares em alunas de nutrição do Rio Grande do Sul. Rev Nutr. 2009;22(2):219-27.

43. Fortes LS, Amaral ACS, Almeida SS, Ferreira MEC. Efeitos de variáveis psicológicas, morfológicas e sociodemográficas sobre o comportamento alimentar de adolescentes. Rev Paul Pediatr. 2013;31(2):182-8.

44. Bighetti F. Tradução e validação do Eating Attitudes Test (EAT-26) em adolescentes do sexo feminino na cidade de Ribeirão Preto-SP [dissertação]. Ribeirão Preto: Universidade de São Paulo;2003.

45. Drescher D. Identificação de sinais e sintomas característicos de anorexia e bulimia nervosas em acadêmicas no ensino superior comunitário [monografia]. Santa Cruz do Sul: Universidade de Santa Cruz do Sul; 2005.

46. Schomer EZ, Kachani AT. Imagem corporal. In: Cordás TA, Kachani AT. Nutrição em psiquiatria. Porto Alegre: Artmed; 2010.

47. Kretschmer AC, Rodrigues G0, Ristow AS, Peixoto NC. Estado nutricional e hábitos alimentares de acadêmicos de uma universidade do norte do Rio Grande do Sul. Rev Saúde (Santa Maria). 2015;41(2):121-8.

48. Feitosa EPS, Dantas CAO, Andrade-Wartha ERS, Marcellini OS, Mendes-Netto RS. Hábitos alimentares de estudantes de uma universidade pública no Nordeste, Brasil. Alim Nutr. 2010;21(2):225-30 
49. Alvarenga MS, Philippi ST, Lourenço BH, Sato PM, Scagliusi FB. Insatisfação coma imagem corporal em universitárias brasileiras. J Bras Psiquiatr. 2010;59(1):44-51.

50. Kravchychyn ACP, Silva DF, Machado FA. Relação entre estado nutricional, adiposidade corporal, percepção de autoimagem corporal e risco para transtornos alimentares em atletas de modalidades coletivas do gênero feminino. Rev Bras Educ Fís Esporte. 2013;27(3):459-66.

51. Bittencourt LJ, Almeida RA. Transtornos alimentares: patologia ou estilo de vida? Psicol Soc. 2013;25(1):220-9.

52. Quadros TMB, Gordia AP, Martins CR, Silva DAS, Ferrari EP, Petroski EL. Imagem corporal em universitários: associação com estado nutricional e sexo. Motriz. 2010;16(1):78- 85.

53. 53 Bosi MLM, Luiz RR, Uchimura KY, Oliveira FP. Comportamento alimentar e imagem corporal entre estudantes de educação física. J Bras Psiquiatr. 2008;57(1):28-33.
54. Souza AA, Souza JC, Hirai ES, Luciano HA, Souza N. Estudo sobre a anorexia e bulimia nervosa em universitárias. Psic Teor Pesq. 2011;27(2):195-8.

55. Marconato MSF, Silva GMM, Frasson TZ. Hábito alimentar de universitários iniciantese concluintes do curso de nutrição de uma universidade do interior paulista. Rev Bras Obes Nutr Emagrecimento. 2016;10(58):180-8.

56. Legnani RFS, Legnani E, Pereira EF, Gasparotto GS, Vieira LF, Campos W. Transtornos alimentares e imagem corporal em acadêmicos de Educação Física. Motriz.2012;18(1):84-1.

57. Vitolo MR, Bortolini GA, Horta RS. Prevalência de compulsão alimentar entre universitárias de diferentes áreas de estudo. Rev Psiquiatr Rio Gd Sul. 2006;28(1):20-6.

58. Martins CR, Petroski EL. Insatisfação com a imagem corporal em adolescentes do sexo feminino de uma cidade de pequeno porte: prevalência e correlações. Motri. 2015;11(2):94-106. 